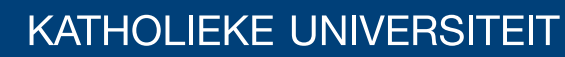 \\ LEUVEN
}

Faculty of Business and Economics

1 HQRUNDQGIFRQNDFWRSWP 4 DURQIIRU

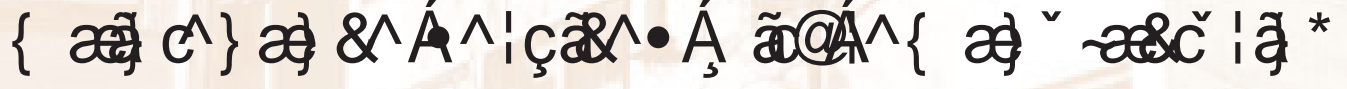

. UNVI IFFHQVII IHANU\&RQQQIO DIFI/ DP EUHFKW

DEPARTMENT OF DECISION SCIENCES AND INFORMATION MANAGEMENT (KBI) 


\title{
Network and Contract Optimization for Maintenance Services with Remanufacturing
}

\author{
Kris Lieckens ${ }^{* 1}$, Pieter Colen ${ }^{\dagger 1}$, and Marc Lambrecht ${ }^{\ddagger 1}$ \\ ${ }^{1}$ Faculty of Business and Economics, Research Center for Operations Management, \\ KU Leuven, Belgium
}

December 4, 2012

\begin{abstract}
Comprehensive long term maintenance contracts are a recent trend in equipment industries. At the same time increasing environmental concerns and high prices for raw materials stress the importance of remanufacturing activities. Hence, maintenance contracts often result in remanufacturing of some critical components. We consider the situation in which an industrial equipment manufacturer offers multi-year maintenance contracts with an uptime guarantee. To provide these service contracts profitably, the company needs to decide on the contract design: selling price, overhaul interval and uptime guarantee. Moreover, the company has to invest in the required logistics network. The logistics network is determined by the locations, number and capacity level of the remanufacturing facilities and the number of field technicians in each service region. The network design and the overhaul interval affect the level of service in terms of machine uptime, and consequently, the price that customers are willing to pay for the service contract. The lead times throughout the network are stochastic in nature

*kris.lieckens@kuleuven.be (corresponding author)

†pieter.colen@kuleuven.be

†marc.lambrecht@kuleuven.be
\end{abstract}


and modeled with steady-state queueing equations. We formulate a non-linear, mixed integer model to optimize the contract and logistics network with the objective of profit maximization for the service provider. The model is applied in a real-life case study and solved by means of a differential evolution algorithm.

Keywords: Contract Design, Remanufacturing, Network Design, Queueing

\section{Introduction}

For most equipment such as trains, elevators, oil-platforms or airplanes, maintenance costs are several times larger than the purchasing cost of the equipment. As customers focus on lifecycle cost and machine uptime, original equipment manufacturers (OEM) are responding with more comprehensive after sales support contracts. These contracts stipulate that the OEM guarantees, against a yearly fixed fee, to perform all corrective and preventive maintenance and to deliver the required parts. Stringent uptime guarantees are often included in these contracts. The increasing importance of such maintenance contracts has significant repercussions for manufacturing companies. Not only do they need to reevaluate their logistics network in order to fulfill their contractual commitments, the contracts also change the incentives for both parties (Kim et al., 2007). As both parties are to gain by avoiding breakdowns, the incentives are more aligned than before: the OEM may change its preventive maintenance policy to avoid costly failures. However, as the financial risk of extensive maintenance cost shifts to the OEM, the company has to be aware of adverse selection and moral hazard risks. The yearly fixed fee may attract primarily customers who use their equipment in extreme conditions (adverse selection). Moreover, being fully covered for any breakdown costs may induce customers to take less care and even neglect their equipment (moral hazard).

Our research effort is inspired by observing the pervasive effect of comprehensive service contracts at a leading manufacturer of industrial equipment. Although this company, which we refer to as AirGen, is successfully selling multi-year service contracts, it is only starting to include uptime guarantees. Therefore, as for many other companies, there is a need to understand the consequences of adding service guarantees. More generally, how does the service contract design (price, uptime guarantee and mainte- 
nance policy) influence the operations (logistics network) and which factors influence the optimal service contract design?

Designing a service contract often boils down to setting a price and a guaranteed machine uptime (or machine availability) (So and Song, 1998; Pangburn and Stavrulaki, 2008). Clearly, the pricing and uptime decisions are related: customers are often willing to pay a premium for fast service (Ray and Jewkes, 2004). High machine availability can only be delivered when sufficient resources (inventory and technicians) are available to repair machines quickly. Although preventive maintenance may limit the number of failures, machines are unavailable during maintenance. Hence, the contracted downtime is not only influenced by capacity decisions, but also by the maintenance policy. Yeh et al. (2009), Yeh et al. (2011) and Pongpech and Murthy (2006) optimize the maintenance policy for leased equipment. Robotis et al. (2012) optimize the price and duration of a leasing contract, while taking into account maintenance actions and remanufacturing possibilities. Bollapragada et al. (2007) set the optimal part replacement interval for a population of machines with remanufacturing of the removed parts. Murthy and Yeung (1995) take a game theoretic approach to study the equilibrium where a customer decides on the maintenance policy taking into account the price setting of a maintenance service provider.

AirGen, the case study company, offers a multi-year comprehensive service contract referred to as a full responsibility contract (FRC). According to this contract type, AirGen guarantees a certain uptime level on the equipment it maintains against a yearly fixed fee. Hence, AirGen takes responsibility for both preventive maintenance and repairs in order to meet the uptime guarantee on their customers' machine. In the case of AirGen, preventive maintenance involves some minor regular interventions, e.g. oil changing, as well as some larger, but less frequently performed overhauls. In this work we only focus on overhauls because they are more expensive. Key for both the FRC profitability and the realized machine uptime is the overhaul interval, i.e. the time between consecutive overhauls. Consequently, the overhaul interval is an important element in the FRC design. In addition to FRC, AirGen also offers ad hoc service for those customers that do not require a comprehensive service contract. Service is then only provided after a customer has placed an order for maintenance, either an overhaul or a repair. This intervention will be invoiced based on the hours and parts consumed to execute this service order. Obviously, FRCs create a long term relationship with AirGen, whereas ad hoc orders are transactional. Thus, customers have three 
options: buy a full responsibility contract, buy ad hoc service or do not buy any service from AirGen (no service option). Depending on customers' uptime and price sensitivities, the different options will differ in popularity.

To accommodate demand for repair and overhauls at the customer sites, the company has to invest in field technicians. We assume that different groups of technicians serve the two customer types (FRC and ad hoc customers). Machine uptime depends on the number of overhauls and failures, but also on the respective process times and variabilities. Especially, the machine repair times (including waiting and traveling time) of the field technicians are key to ensure a certain uptime. It is clear that waiting time created by unavailability of field technicians to repair a failed machine constitutes downtime for the machine. The durations of the waiting time depend on the utilization level of the technicians, which is the result of confronting the workload with the available capacity.

A complicating factor in our study is that during a machine overhaul the key component of the equipment is replaced by an as good-as-new part. This replacement part can be either a remanufactured or a newly produced one. The removed used part is sent to a specialized remanufacturing center to be remanufactured, after which it can serve as a replacement part for another overhaul. Clearly, the OEM should ensure that a sufficient amount of already remanufactured parts is available in stock. Since this stock level depends on the lead times and the uncertainty in the network, factors that influence these measures should be taken into account while designing service contracts. These factors include the location and capacity of remanufacturing centers and total variability of process and demand characteristics.

Given the importance of lead times to achieve the uptime guarantee and to determine the inventory stock level, we use an advanced queueing model with steady state relationships to estimate how much the OEM has to invest in stock of already remanufactured parts and field technician capacity to maintain the machines such that a given machine uptime is guaranteed. Given the complex interrelationships between contract characteristics and network design decisions, we prefer to optimize the integrated contract and network design model with concurrent decisions on facility locations, capacity and FRC features (yearly fixed fee, overhaul interval and machine uptime guarantee).

Figure 1 gives an overview of the AirGen network under consideration. Field technicians are located in 
a dedicated service region, i.e. they only execute orders from their own region. However, in each region two types of technicians are considered: technicians for FRC and for ad hoc customers. Although this distinction in field capacity is not needed from a modeling perspective, we prefer to work with two types of field technicians to capture the difference in treatment for the two customer groups. To execute an overhaul, field technician capacity should be available in the service region. Next, the technician who executes the overhaul replaces the used part by a remanufactured one. The used component is sent to one of the six potential remanufacturing facilities. When there is insufficient supply of used parts, new parts are delivered by the manufacturing plant. Instead of immediately replacing the used part by another, already remanufactured one, an alternative overhaul strategy could be to re-install the original part after it has finished the complete remanufacturing process. Lieckens et al. (2012) compare these two strategies and conclude that immediate replacement of a used part by an already remanufactured one is the most cost efficient practice. Consequently, the model in this study only considers that overhaul strategy.

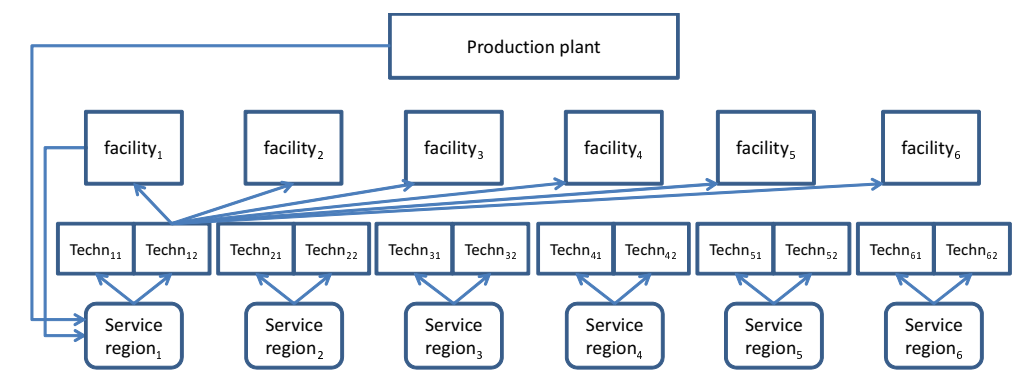

Figure 1: The case study network

To summarize, the goal of this research is to investigate the consequences of contract selling in aftermarket services taking into account the interplay between market dynamics, pricing, maintenance and network optimization. Based on queuing relationships and reliability modeling, it is possible to quantify the impact of the investment decisions in capacity, inventory and facility locations on the uptime of the machines being maintained. The resulting uptime, together with the price, will influence demand and hence the workload. Investment decisions need to be made to accommodate this workload. The model contains a demand sub-model (price, uptime guarantee and overhaul interval) and a logistics network sub-model (facility location, capacity and inventory). The two sub-models and the profit objective function are explained in the next section. Section 3 presents our main findings and insights. We conclude in Section 4. 


\section{Mathematical Model}

In this section, we build the profit maximization model, which consists of a demand sub-model and a network sub-model. The objective is profit maximization in steady-state for a one year time interval (all time measures are expressed in years). Whereas the demand sub-model primarily deals with the contract design (Section 2.1), the network optimization sub-model ensures that the conditions in the contract can be delivered by making appropriate investment decisions (Section 2.2). The two sub-models are combined in a mixed integer non-linear model, which is solved by the differential evolution method as an improved genetic search algorithm (Section 2.3). The model formulation relies on five groups of decision variables whose notation will be explained in the following sections:

1. binary variables $Y_{r}$ to decide on the opening and closing of remanufacturing centers (facility location problem)

2. integer variables to decide on the number of field technicians $s_{t}$ and the number of remanufacturing operators $s_{r}$ (capacity problem)

3. continuous variables $\varpi_{c s}$ to decide on the yearly number of different contract types (demand submodel)

4. continuous variables $T O_{c 1}$ to decide on the overhaul interval for FRC customers only (demand sub-model)

5. continuous variables $\tau_{c s r}$ to assign a proportion of the used parts flow to a remanufacturing center $r$ (logistics network sub-model)

Since inventory levels for parts are dependent on their aggregate lead time and this lead time is driven by all the variables mentioned above, no additional decision variables are required to estimate the number of units in stock (see Section 2.2.3). Figure 2 represents the logic of the model: the most critical parameters, decision variables and the interdependent relationships. It is clear that both sub-models (for the demand and the logistics network) are connected through the demand for maintenance jobs and the lead time to execute maintenance jobs. Note that the overhaul for the ad hoc service is a parameter with given value, while it is a decision variable for FRC service. 


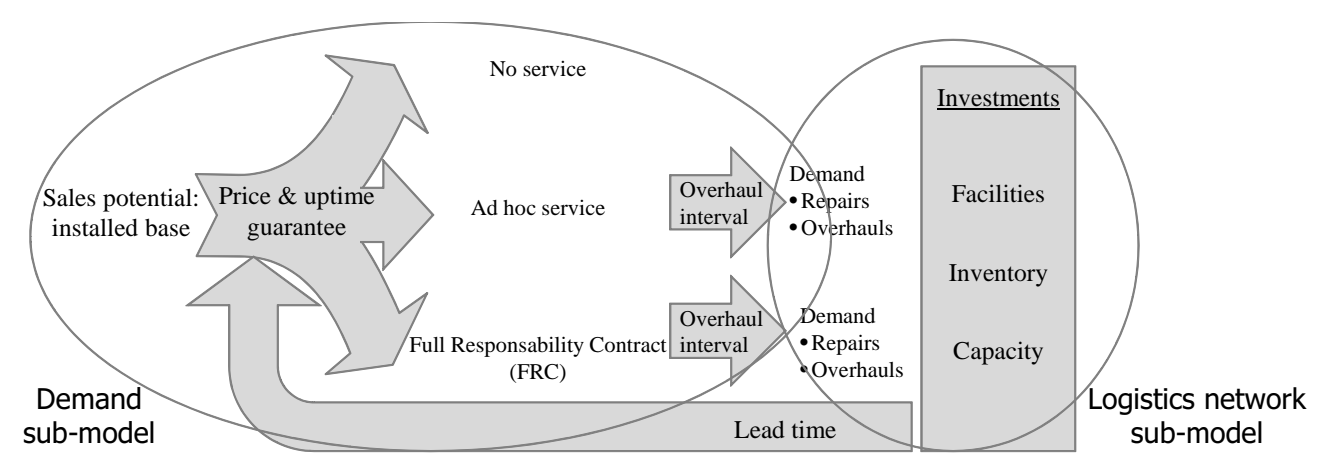

Figure 2: Parameters, variables and relationships in the model

\subsection{Demand Sub-Model}

In this section, we characterize the demand for each service option depending on the service contract design. Demand for service generates flows in the queueing network that can be characterized by the expected arrival rates and the variability of interarrival times, which are expressed as a squared coefficient of variation $(\mathrm{SCV})$.

In our case study, customers have the possibility to choose from three service products $s=\{1, \ldots, S=3\}$ : FRC service $(s=1)$, ad hoc service $(s=2)$ or no service from AirGen $(s=3)$. Each customer belongs to one of the six service regions $c=\{1, \ldots, C=6\}$, where the service product $s$ is offered against a region specific, yearly fixed selling price $S P_{c s}$ and with an overhaul interval $T O_{s}$ that is the same in all regions $c$. The latter can easily be relaxed towards region specific overhaul intervals. The potential (or maximum) demand in each region $c$ for FRC, i.e. $D_{c 1}$, is equal to its installed base $D_{c}^{\max }$. Remember that ad hoc service occurs when the customer does not buy a comprehensive service contract but orders service interventions whenever necessary. This type of demand can be for both repair and overhaul interventions. The third service option corresponds to a "no service order" decision from the customer. To quantify the volume for each service product $s$, i.e. estimating the probability of choosing a certain option, we apply a multinomial logit model for each service region. These models quantify the probability that a customer chooses a certain option within a given set of choices. Moreover, multinomial logit models offer the benefit of easy estimation of the demand sensitivities based on conjoint choice surveys (Vermeulen et al., 2011; Haaijer et al., 2001). There are three choice probabilities, either a customer in region $c$ buys an FRC with probability $P_{c 1}$, or only orders service as deemed necessary with probability $P_{c 2}$, or opts for no 
AirGen service with the remaining probability $P_{c 3}$. We write the multinomial logit model for region $c$ as follows:

$$
\begin{aligned}
P_{c s} & =\frac{\varpi_{c s}}{\sum_{s=1}^{S} \varpi_{c s}} \\
= & \frac{e^{\varepsilon_{S P_{c}} S P_{c s}+\varepsilon_{D T_{c}} D T_{c s}}}{\sum_{S=1}^{S} e^{\varepsilon_{S P_{c}} S P_{c s}+\varepsilon_{D T_{c}} D T_{c s}}}
\end{aligned}
$$

with $P_{c s}=$ probability of buying FRC $(s=1)$, ad hoc service $(s=2)$

$$
\begin{aligned}
& \text { or no service }(s=3) \text { in region } c \\
& \varepsilon_{S P_{c}}=\text { price sensitivity in region } c \\
& S P_{c s}=\text { yearly price for } \operatorname{FRC~}(s=1) \text {, ad hoc service }(s=2) \text { or } \\
& \text { no service }(s=3) \text { in region } c \\
& \varepsilon_{D T_{c}}=\text { downtime sensitivity in region } c \\
& D T_{c s}=\text { average downtime with FRC }(s=1) \text {, ad hoc service } \\
& (s=2) \text { or no service }(s=3) \text { in region } c
\end{aligned}
$$

This demand characterization is useful in our model because we are interested in the price that simultaneously corresponds to a volume decision on the one hand and to the downtime resulting from a particular setting for the five decision variables on the other hand.

Based on the demand sensitivities, the market reaction on prices and availability guarantees can be estimated. Machine availability, or uptime, for service $s$ in region $c$ is directly related to its downtime $D T_{c s}$ ( $D T_{c S}$ is expressed in years)

$$
\text { Availability }_{c s}=1-D T_{c s}
$$

Once the probabilities to select one of the options are known in region $c$, its yearly expected demand for 
the different service products $\left(D_{c s}\right)$ can be derived from the size of the installed base $\left(D_{c}^{\max }\right)$ :

$$
D_{c s}=D_{c}^{\max } P_{c s}
$$

Expected revenue is given by

$$
R E V=\sum_{c=1}^{C} \sum_{s=1}^{S-1} D_{c s} S P_{c s}
$$

From Equations 1 and 2, it is clear that there are three unknown variables in the demand function: $D_{c s}$, $D T_{c s}$ and $S P_{c s}$. The downtime $D T_{c s}$ which will be derived in Equation 16, depends on the lead times. However these lead times depend on the total volume (demand) that needs to be processed. The demand level $D_{p}$ on his turn depends on the realized downtime $D T_{c s}$ and the prices of the service options $S P_{c s}$. Therefore we treat each $\bar{\omega}_{c s}$ in Equation 1 as a decision variable which allows to calculate the demand volume $\left(D_{c s}\right)$. Due to negative elasticity parameters, values for these variables must be chosen in the interval $[0,1]$. Once the volume is known the downtime can be determined. Next, the resulting price is a function of both $\varpi_{c s}$ and $D T_{c s}$. After reorganizing Equation 1, the selling price is given by

$$
S P_{c s}=\frac{\ln \left(\Phi_{c s}\right)-\varepsilon_{D T_{c}} D T_{c s}}{\varepsilon_{S P_{c}}}
$$

The volume of FRC and ad hoc services sold determines the amount of work to be processed in remanufacturing centers and by field technicians. In each service region, both service contract and ad hoc customers require overhauls $\lambda_{c s}$ and repair service $\kappa_{c s}$. We express these demand levels as flows (demand for interventions per year). As the logistics network relies on a queuing model, we are interested in the corresponding flow rates, interarrival times and their squared coefficients of variation (SCV).

The yearly expected demand for repairs and overhauls depends on the operating hours of the machines. From what we have observed in practice, the yearly operating hours of the machines in the installed base can be characterized by a triangular distribution with parameters $e_{c}, f_{c}$ and $g_{c}$ for the lower bound, upper bound and maximum, respectively. The distribution of the demand for overhauls is retrieved by first dividing the triangular distribution of the yearly operating hours by the overhaul interval $T O_{s}$, also 
expressed in operating hours, followed by multiplication with the total number of service products $D_{c s}$. This results in a new triangular distribution with lower bound $e_{c s}=\left(e_{c} / T O_{s}\right) D_{c s}$, upper bound $f_{c s}=$ $\left(f_{c} / T O_{s}\right) D_{c s}$ and maximum $g_{c s}=\left(g_{c} / T O_{s}\right) D_{c s}$. This represents the distribution of the yearly demand for overhauls with an average rate of $\lambda_{c s}=\left(e_{c s}+f_{c s}+g_{c s}\right) / 3$ and variance $\sigma_{D_{c s}}^{2}=\left(e_{c s}^{2}+f_{c s}^{2}+g_{c s}^{2}-\right.$ $\left.e_{c s} f_{c s}-e_{c s} g_{c s}-f_{c s} g_{c s}\right) / 18$. For machines under FRC, $\lambda_{c 1}$ depends on the overhaul interval $T O_{1}$, a parameter that must be decided upon by AirGen. Consequently, $T O_{1}$ is a decision variable in our model. On the other hand, for overhauls originating from ad hoc customers $\left(\lambda_{c 2}\right)$, the overhaul interval is set by customers themselves, typically equal to the maximal value as prescribed by the machine maintenance specifications. Hence, $\mathrm{TO}_{2}$ is a given parameter in our model.

To derive the SCV of the time between overhauls, i.e. $c_{I A(\lambda)_{c s}}^{2}$, we rely on the relationships $E[1 / Y]=$ $1 / E[Y]\left(1+\operatorname{Var}[Y] / E[Y]^{2}\right)$ and $\operatorname{Var}[1 / Y]=(1 / E[Y])^{2}\left(\operatorname{Var}[Y] / E[Y]^{2}\right)$ with $Y$ the number of overhauls per year. The distribution of the interarrival time of overhauls from service product $s$ has an average number of years equal to $I A(\lambda)_{c s}=\left(1 / D_{c s}\right)\left(1+\sigma_{D_{c s}}^{2} / D_{c s}^{2}\right)$ with a variance equal to $\sigma_{I A(\lambda)_{c s}}^{2}=\left(1 / D_{c s}\right)^{2}\left(\sigma_{D_{c s}}^{2} / D_{c s}^{2}\right)$. This results into $c_{I A(\lambda)_{c s}}^{2}=\left(\sigma_{I A(\lambda)_{c s}} / I A(\lambda)_{c s}\right)^{2}$.

In addition to the demand for overhauls, we need to characterize the demand for repair service. The yearly number of failures depends on the machine reliability, yearly operating hours and the overhaul interval. We assume that a repair brings a machine back in operating condition but without altering its failure rate (minimal repair). Overhauls, however, rejuvenate the machine to an as-good-as-new condition. For repairable systems the Power Law process is the most widely applied reliability model. For a Power Law process, the intensity function $h(t)$ that describes the evolution of the failure rate can be written as

$$
\begin{aligned}
h(t) & =\frac{\beta}{\alpha}\left(\frac{t}{\alpha}\right)^{\beta-1} \\
& =\operatorname{PL}(\alpha, \beta),
\end{aligned}
$$

with $\alpha=$ scale parameter

$\beta=$ shape parameter

$t=$ cumulative number of operating hours since last overhaul

Note that the machine reliability as defined here is with one set of $\alpha$ and $\beta$ values. This is for notational 
convenience only, the model can have different $\alpha$ and $\beta$ values depending on the region $c$ and/or service product $s$. The expected number of failures between two consecutive overhauls equals

$$
M\left(T O_{s}\right)=\int_{0}^{T O_{s}} h(t) d t=\left(\frac{T O_{s}}{\alpha}\right)^{\beta}
$$

When this equation is multiplied by the average number of overhauls per year, we find the yearly average arrival rate of failures of machines in region $c$ with service product $s$ :

$$
\kappa_{c s}=\left(T O_{s} / \alpha\right)^{\beta} \lambda_{c s}
$$

To find the SCV of the interarrival times of failures expressed in years, i.e. $c_{I A(\kappa)}^{2}$, we rely on the relationship

$$
\operatorname{Var}[X Y]=E[Y] E[Y] \operatorname{Var}[X]+E[X] E[X] \operatorname{Var}[Y]+\operatorname{Var}[X] \operatorname{Var}[Y]
$$

with $X$ the time between overhauls expressed in years and $Y$ the time between failures expressed in overhaul periods. $E[X]$ and $\operatorname{Var}[X]$ have been derived as $I A(\lambda)_{c s}$ and $\sigma_{I A(\lambda)_{c s}}^{2}$, while $Y$ is exponentially distributed with parameter $\left(T O_{S} / \alpha\right)^{\beta}$. This leads to

$$
c_{I A(\kappa)_{c s}}^{2}=\frac{\left(1 /\left(T O_{s} / \alpha\right)^{\beta}\right)^{2}\left(2 \sigma_{I A(\lambda)_{c s}}^{2}+I A(\lambda)_{c s}^{2}\right)}{\left(1 / \kappa_{c S}\right)^{2}}
$$

Now, we have characterized the demand for overhaul and repair for each of the service products $s$ in each customer region $c$. We assume that for each $c$-s combination, except for the no service option $s=S$, there is a unique, dedicated team of field technicians $t \in\{1, \ldots, T=C \times(S-1)=12\}$ that will experience the demand for repair and overhaul service. We use $\zeta_{c s t}=1$ if technician $t$ is assigned to maintain service product $s$ in region $c$ and $\zeta_{c s t}=0$ otherwise. Since each technician $t$ is exclusively assigned to one $c$ $s$ combination, there exists only one technician $t$ where $\zeta_{c s t}=1$ for a specific $c$-s combination. From $s$-machines in $c$-regions, they will experience an expected arrival rate of overhauls and corresponding 
emergency failures of respectively

$$
\lambda_{t}=\sum_{c=1}^{C} \sum_{s=1}^{S-1} \varsigma_{c s t} \lambda_{c s} \quad \kappa_{t}=\sum_{c=1}^{C} \sum_{s=1}^{S-1} \varsigma_{c s t} \kappa_{c s}
$$

The aggregate arrival rate of service jobs for technicians $t$ becomes $\psi_{t}=\lambda_{t}+\kappa_{t}$. For its SCV of aggregate interarrival times, we use the approximation from Lambrecht et al. (1998)

$$
c_{I A_{t}}^{2} \approx \frac{1}{3}+\frac{2}{3}\left(\sum_{c=1}^{C} \sum_{s=1}^{S-1} \zeta_{c s t}\left(\lambda_{c s} c_{I A(\lambda)_{c s}}^{2}+\kappa_{c s} c_{I A(\kappa)_{c s}}^{2}\right) / \psi_{t}\right)
$$

\subsection{Logistics Network Sub-Model}

We consider a two echelon network in which the first echelon consists of field technicians $t$. Each service region has technicians dedicated to FRC and ad hoc customers. The second echelon contains remanufacturing centers $r \in\{1, \ldots, R\}$ for remanufacturing parts removed during an overhaul and manufacturing plants $n \in\{1, \ldots, N\}$ as an alternative source for parts. In our model for AirGen (Figure 1), there are six potential remanufacturing centers to choose from $(R=6)$ and one production facility $(N=1)$ that is always open.

In order to find the flows in the network, components from machines in region $c$ that are under service option $s \in\{1, \ldots, S-1\}$ are assigned to a remanufacturing facility $r$ according to the proportion $\tau_{c s r}$. If the assumption applies that no units are discarded by the field technicians, then the yearly average arrival rate of these parts at facility $r$ is $\lambda_{c s r}=\tau_{c s r} \lambda_{c s}$, subject to the constraint that the volume to be remanufactured is not allowed to exceed the demand for overhauls (number of removed parts):

$$
\tau_{c s}=\sum_{r=1}^{R} \tau_{c s r} \leq 1 \quad c=1, \ldots, C \text { and } s=1, \ldots, S-1
$$

Inequality (5) allows for accepting less parts in the system than the maximum yearly volume of removed parts $\left(\lambda_{c s}\right)$. This may occur if it is more economic to provide new parts instead of delivering remanufactured ones. This is possible when the lead times, the inventory levels, the downtime and the selling price, and thus also the demand for service products, can benefit from the reduced workload at remanufacturing 
centers in such a way that the additional cost of producing new ones is compensated. Expected aggregate arrival rate per year at a facility $r$ becomes $\lambda_{r}=\sum_{c=1}^{C} \sum_{s=1}^{S-1} \lambda_{c s r}$ with a SCV $c_{I A_{r}}^{2}$ to be derived in Equation 14 . Due to quality problems identified after the remanufacturing process, some parts will be disposed of at a rate $\omega_{c s r}$. We assume that there is no scrap in the production of new parts $\left(\omega_{c s n}=0\right)$. Since all demand for overhauls must be satisfied, the single production plant $(n=1)$ must deliver the total yearly average net requirement of new parts $\lambda_{c s n}=D_{c s}-\sum_{r=1}^{R}\left(1-\omega_{c s r}\right) \lambda_{c s r}$ for machines in each customer region $c$ that are under service option $s \in\{1, \ldots, S-1\}$. Since we assume infinite capacity at this plant, the SCV of the interarrival times is given as $c_{I A_{n}}^{2}$.

At this point, all the information is given to discuss the three problems in the logistics network sub-model, i.e. the facility location, the capacity and the inventory problem.

\subsubsection{Facility Location Problem}

Given the flow rates that result from the demand sub-model (section 2.1), we first define the cost trade-offs related to the opening of remanufacturing centers $r$ chosen from a predefined set of potential locations $r \in$ $\{1, \ldots, R\}$. The binary decision variable $Y_{r}$ is used to either open or close a location $r$. The fixed facility cost $F C$, total transportation cost $T C$ and total process cost $P C$ are important drivers in the selection process of remanufacturing centers:

$$
\begin{aligned}
F C= & \sum_{t=1}^{T} F C 2_{t} s_{t}+\sum_{r=1}^{R}\left(F C 1_{r}+F C 2_{r} s_{r}\right) Y_{r} \\
T C= & \sum_{t=1}^{T} \psi_{t} U T C_{t}+ \\
& \sum_{c=1}^{C} \sum_{s=1}^{S-1}\left(\sum_{r=1}^{R} \lambda_{c s r}\left(2-\omega_{c s r}\right) U T C_{c s r}+\sum_{n=1}^{N} \lambda_{c s n} U T C_{c s n}\right)
\end{aligned}
$$




$$
\begin{aligned}
P C= & \sum_{t=1}^{T}\left(\lambda_{t} U P C_{t}+\kappa_{t} U P C_{t}^{\prime}\right)+\sum_{c=1}^{C} \sum_{s=1}^{S-1}\left(\sum_{n=1}^{N} \lambda_{c s n} U P C_{c s n}+\right. \\
& \left.\sum_{r=1}^{R} \lambda_{c s r}\left(U P C_{c s r}+\omega_{c s r} U D C_{c s r}\right)+\left(1-\tau_{c s}\right) \lambda_{c s} U D C_{c s}\right)
\end{aligned}
$$

For the yearly fixed facility cost $F C$ in Equation 6, we have a fixed overhead cost $F C 1_{r}$, which is charged for e.g. depreciation cost, administration and management overhead costs. Moreover, also a fixed cost for each field technician $\left(F C 2_{t} s_{t}\right)$ and remanufacturing operator $\left(F C 2_{r} s_{r}\right)$ is charged. When a facility $r$ is closed, the flow of parts is non-existent, i.e. $\tau_{c s r}=0$. All transportation activities in the network are performed by third party logistics companies (3PL), that ship on a one by one basis (lot size=1). The total yearly transportation cost $T C$ in Equation 7 depends on a unit tariff for the technician traveling to the customer $\left(U T C_{t}\right)$, the transportation of the part from the customer site to a remanufacturing center and back (twice the tariff $U T C_{c s r}$, excluding scrap at remanufacturing centers), and the transportation cost between the customer site and the production plant $\left(U T C_{c s n}\right)$. These unit costs are multiplied with their respective yearly volumes. The production cost $P C$ accounts for the unit processing cost of the technicians for overhauls and repairs $\left(U P C_{t}\right.$ and $\left.U P C_{t}^{\prime}\right)$, unit processing cost at remanufacturing $\left(U P C_{c s r}\right)$ including the unit disposal cost $\left(U D C_{c s r}\right)$, unit processing cost at the plant for producing new parts $\left(U P C_{c s n}\right)$ and disposal cost for units that are not allowed into the network $\left(U D C_{c s}\right)$.

\subsubsection{Capacity Problem}

Total time that parts spend in the network, also referred to as lead time or throughput time, is a critical performance measure in our model. It determines the downtime of machines (and hence selling prices of the services) and the required inventory levels to satisfy given inventory service levels defined as the minimal percentage of parts to be delivered directly from stock. In order to achieve an appropriate lead time, we need to set appropriate capacity levels for technicians and remanufacturing operators, represented by respectively $s_{t}$ and $s_{r}$. To this end, we transform the network into a queueing system using steady state equations. It consists of arrival processes as described in Section 2.1 and various processing steps (field maintenance, remanufacturing, production of new parts and transportation). As a result, 
we can decide on the number of technicians and remanufacturing operators while taking into account endogenously determined volumes and different sources of variability. Because of their constrained capacity, queues arise at the field technicians $t$ and remanufacturing facilities $r$, while the assumption of infinite capacity at the 3PL and the production plant leads to empty queues there. Since the input processes are clearly not Markovian and all processing times, including transportation, are assumed to be generally distributed, we model all inbounds as $\mathrm{G} / \mathrm{G} / \mathrm{m}$ queues.

In order to establish the relationship between capacity levels and lead times, we need to collect process data to derive the following effective process time characteristics, expressed in years: mean overhaul time $P R_{t}$ and mean repair time $P R_{t}^{\prime}$ for technicians $t$ (with variances $\sigma_{P R_{t}}^{2}$ and $\sigma_{P R_{t}^{\prime}}^{2}$, and SCV $c_{P R_{t}}^{2}$ and $c_{P R_{t}^{\prime}}^{2}$ ), mean remanufacturing time $P R_{c s r}$ (with variance $\sigma_{P R_{c s r}}^{2}$ and $\operatorname{SCV} c_{P R_{c s r}}^{2}$ ), and mean production time for new items $P R_{c s n}$ (with variance $\sigma_{P R_{c S n}}^{2}$ and $\operatorname{SCV} c_{P R_{c s n}}^{2}$ ).

These processing time characteristics in terms of mean and variance expressed in years do not only differ according to the service type $s$ due to e.g. different administrative formalities, also the region $c$ may impact these characteristics due to e.g. different working conditions. In our stochastic model, we use effective measures for these characteristics by taking into account any disruption of technicians and operators, e.g. their non-value added working time (training, lunch, break, ...), absenteeism and other efficiency losses. Given a lot size equal to one for all process steps, set-up times that possibly exist, are included in the effective processing time.

When multiple parts $s$ arrive from multiple regions $c$, queue time estimation at technicians $t$ and remanufacturing centers $r$ requires aggregation of their processing characteristics into a single value by using a weighted average. As technicians $t$ have to deal with both overhauls and repairs, the average process time at technicians $t$ and its SCV equal

$$
\begin{aligned}
& P R_{t}=1 / \mu_{t}=\left(\lambda_{t} P R_{t}+\kappa_{t} P R_{t}^{\prime}\right) / \psi_{t} \\
& c_{P R_{t}}^{2}=\frac{\mu_{t}^{2}}{\psi_{t}}\left(\lambda_{t} P R_{t}^{2}+\kappa_{t} P R_{t}^{\prime 2}\right)-1+\frac{1}{\psi_{t}}\left(\lambda_{t} c_{t}^{2}+\kappa_{t} c_{t}^{\prime 2}\right)
\end{aligned}
$$


At remanufacturing facilities $r$ average process time and its SCV can be expressed as

$$
\begin{aligned}
& P R_{r}=1 / \mu_{r}=\sum_{c=1}^{C} \sum_{s=1}^{S-1} \sum_{r=1}^{R} \lambda_{c s r} P R_{c s r} / \lambda_{r} \\
& c_{P R_{r}}^{2}=\sum_{c=1}^{C} \sum_{s=1}^{S-1} \sum_{r=1}^{R} \frac{\lambda_{c s r}}{\lambda_{r}} P R_{c s r}^{2} \mu_{r}^{2}-1+\sum_{c=1}^{C} \sum_{s=1}^{S-1} \sum_{r=1}^{R} \frac{\lambda_{c s r}}{\lambda_{r}} c_{c s r}^{2}
\end{aligned}
$$

The utilization level of the technicians $t$ and the operators in the remanufacturing centers $r$ is determined as

$$
\rho_{t}=\lambda_{t} / \mu_{t} s_{t} \leq 1 \quad \rho_{r}=\lambda_{r} / \mu_{r} s_{r} \leq 1
$$

The final input parameter for the queueing network model is the SCV of the aggregate interarrival time at a remanufacturing facility $r$ or $c_{I A(\lambda)_{r}}^{2}$. This can be derived if we know the variability of the interdeparture times of parts leaving technicians $t$. Buzacott and Shanthikumar (1993) have presented several approximations for systems with multiple servers, but we opt for the following linking equation (Hopp and Spearman, 2000):

$$
c_{I D_{t}}^{2} \approx 1+\left(1-\rho_{t}^{2}\right)\left(c_{I A_{t}}^{2}-1\right)+\frac{\rho_{t}^{2}}{\sqrt{s_{t}}}\left(c_{P R_{t}}^{2}-1\right)
$$

It is clear that the transportation processes do not affect this interdeparture process because of the unlimited capacity for transportation.

Next, we use a result from Shanthikumar and Buzacott (1981) for the relationship between $c_{I D_{t}}^{2}$ and the $\mathrm{SCV}$ of the aggregate interarrival time at remanufacturing facility $r$ for units coming from technicians $t$, 
represented by $c_{I A_{t r}}^{2}$

$$
c_{I A_{t r}}^{2}=\pi_{t r} c_{I D_{t}}^{2}+\left(1-\pi_{t r}\right)
$$

This depends on $\pi_{t r}$, the fraction of total flow that is going from technician $t$ to remanufacturing center $r$

$$
\pi_{t r}=\sum_{c=1}^{C} \sum_{s=1}^{S-1} \frac{\lambda_{t} \tau_{c s r}}{\psi_{t}}
$$

According to these authors, the expression for $c_{I A_{r}}^{2}$ is the sum of a weighted average of $c_{I A_{t r}}^{2}$

$$
c_{I A_{r}}^{2}=\sum_{t=1}^{T}\left(\frac{\Psi_{t}}{\lambda_{r}} \pi_{t r}\right) c_{I A_{t r}}^{2}
$$

which can be solved by combining Equations 12 to 13 .

The capacity levels of resources $t$ and $r\left(s_{t}\right.$ and $s_{r}$ ), their utilization levels (Equation 11), and the aggregate variability levels of their arrivals (Equations 4 and 14) and production processes (Equations 9 and 10) all influence their expected queue times. We use the approximation from Whitt (1993)

$$
E W Q_{x} \approx \phi\left(\rho_{x}, c_{I A_{x}}^{2}, c_{P R_{x}}^{2}, s_{x}\right)\left(\frac{c_{I A_{x}}^{2}+c_{P R_{x}}^{2}}{2}\right)\left(\frac{\rho_{x}^{\sqrt{2\left(s_{x}+1\right)}-1}}{s_{x}\left(1-\rho_{x}\right)}\right) P R_{x}
$$

where $x=t$ and $x=r$. It is an accurate $\mathrm{GI} / \mathrm{G} / \mathrm{m}$-model with a correction factor $\phi$ that applies to a system under heavy traffic conditions with multiple parallel servers (Hopp and Spearman, 2000). We refer to Whitt (1993) for more details about this factor $\phi$. Since lead times clearly depend on capacity, the capacity decision can be linked with machine uptime and inventory levels (see below), as well as with contract selling prices. Remember from Equation 1 that total downtime for each machine in region $c$ that is under contract type $s$ is a key driver for the selling price. At this point, all components are known to find the expression for the total expected downtime, expressed in years

$$
D T_{c s}=\sum_{t=1}^{T}\left[\frac{\lambda_{t}}{D_{c s}} P R_{c s t}+\frac{\kappa_{t}}{D_{c s}}\left(E W Q_{t}+T R_{c t}+P R_{c s t}^{\prime}\right)\right]
$$


where $T R_{c t}$ is the expected technician travel time to a customer in region $c$, which is assumed to be the same for all customers in this region. The first component is the downtime caused by overhauling a machine, while the second component is the downtime due to machine failures (repairs). Failures are more severe because apart from technician's process time, the machine is unavailable during the queueing and travel delay of the technician. Total yearly volume of overhauls and failures is divided by $D_{c s}$ (yearly number of contracts) because we need the downtime of an individual machine.

\subsubsection{Inventory Problem}

So far, we have explained the relationships between the facility location and the corresponding costs on the one hand and the capacity decisions and lead times on the other hand. The next step is to determine the inventory levels across the network. There are two types of inventory: work-in-process and safety stock at the outbound of remanufacturing and production plants to avoid shortages. The latter is inventory of asgood-as-new parts ready to be shipped directly to the customer when a technician performs an overhaul. This part replaces the used part in the machine at the customer site, while the used part is sent through the remanufacturing cycle to replenish the safety stock. A safety stock of new parts is required for scrapped units. So each time a unit is taken from stock, it will be replenished by either a used or a new part. Consequently, the inventory is managed according to a continuous review, one-for-one replenishment policy, i.e. an $(S-1, S)$ inventory system where $S$ is the order-up-to level.

Based on a target probability for available stock of critical parts at facilities $r$ and $n$, expressed as probability $x_{r} \%$ and $x_{n} \%$, we derive the appropriate order-up-to levels $S_{r}$ and $S_{n}$ in a similar way as Nozick and Turnquist (2001). These levels are composed of two components: inventory to satisfy the expected demand during total lead time, further referred to as $\hat{\mu}_{D D L T}$, and additional inventory to satisfy a given inventory service level, i.e. safety stock $\hat{S S}: S=\hat{\mu}_{D D L T}+\hat{S S}$. In order to find these components, we need the aggregate lead time at remanufacturing center $r$ and production plant $n$, as well as the distribution of the demand during lead time (DDLT).

We first determine for each $c$-s combination the total expected lead time (replenishment lead time) and

its variance, both for remanufactured $\left(L T_{c s r}, \sigma_{L T_{c s r}}^{2}\right)$ and newly produced items $\left(L T_{c s n}=P R_{c s n}, \sigma_{L T_{c s n}}^{2}=\right.$ 
$c_{P R_{c s n}}^{2} P R_{c s n}^{2}$ with $\left.n=1\right)$. For the remanufactured items, we have

$$
\begin{gathered}
L T_{c s r} \approx \sum_{t=1}^{T} \varsigma_{c s t} P R_{t}+T R_{c r}+E W Q_{r}+P R_{c s r}+T R_{c r} \\
\sigma_{L T_{c s r}}^{2} \approx \sum_{t=1}^{T} \zeta_{c s t} \sigma_{P R_{t}}^{2}+\sigma_{T R_{c r}}^{2}+\sigma_{W Q_{r}}^{2}+\sigma_{P R_{c s r}}^{2}+\sigma_{T R_{c r}}^{2}
\end{gathered}
$$

where $\sigma_{T R_{c r}}^{2}$ can be derived from the expected transportation time $T R_{c r}$ and its associated SCV $c_{T R_{c r}}^{2}$, both independent of the service option $s$. We include the transportation process between a customer region $c$ and remanufacturing $r$, upstream as well as downstream, because these delays need to be protected by safety stock. Queueing and transportation delays at the technician are not taken into account because the part arrival at the customer site is coordinated with the arrival of the technician at the site. For the variance of the waiting time at remanufacturing $\sigma_{W Q_{r}}^{2}$, we opt for the approximation from Whitt (1993) (equation 15).

Since the inventory of service parts that is available at the outbound of a facility $r$ or $n$ can be used to serve any demand for refurbished parts, irrespective of the customer region $c$ that has generated the demand and irrespective of the service contract type $s$, the next step is to aggregate these lead times into an aggregate expected and weighted lead time observed at the outbound of each facility $r$ and $n$ with mean $L T_{r}$ and $L T_{n}$, and variance $\sigma_{L T_{r}}^{2}$ and $\sigma_{L T_{n}}^{2}$. We have

$$
\begin{aligned}
& L T_{r} \approx \sum_{c=1}^{C} \sum_{s=1}^{S-1} \frac{\lambda_{c s r}\left(1-\omega_{c s r}\right)}{\lambda_{r}^{\prime}} L T_{c s r} \\
& \sigma_{L T_{r}}^{2} \approx \sum_{c=1}^{C} \sum_{s=1}^{S-1} \frac{\lambda_{c s r}\left(1-\omega_{c s r}\right)}{\lambda_{r}^{\prime}}\left[L T_{c s r}^{2}+\sigma_{L T_{c s r}}^{2}\left(\frac{L T_{r}}{L T_{c s r}}\right)^{2}\right]-L T_{r}^{2}
\end{aligned}
$$

where $\lambda_{r}^{\prime}=\sum_{c=1}^{C} \sum_{s=1}^{S-1} \lambda_{c s r}\left(1-\omega_{c s r}\right)$. We refer to Equation 10 to explain the derivation of these aggregate variances, knowing that e.g. $c_{r}^{2}=\sigma_{L T_{r}}^{2} / L T_{r}^{2}$. Although Equations 17 and 18 are derived for remanufacturing facilities $r$, the same relationships apply for the production facility $n\left(\omega_{c s n}=0\right)$.

The DDLT distribution is a convolution of the demand distribution and the lead time distribution. The demand distribution is triangular due to the triangular distribution of the yearly operating hours of the 
machines. The lead time distribution is assumed to be lognormal (Lambrecht et al., 1998). To find the general, best fit parametric distribution for the DDLT distribution under our modeling assumptions, we conduct a simulation study similar as Lieckens et al. (2012). First, observations are sampled from both a triangular and a lognormal distribution with different parameters. For each scenario, 25000 arrivals are selected from 12 different triangular distributions that are based on triangular running hours with constant lower and upper bound of respectively 4000 and 8000 in combination with three maxima $\in$ $\{5000,6000,7000\}$, four values for running hours between overhauls $\in\{2000,4000,6000,8000\}$ and a constant installed base of 1500. The lognormal lead times are selected from 4 different distributions with a constant mean of 10 days in combination with four standard deviation levels $\in\{1,5,10,15\}$. This results in a total of 48 scenarios. Next, the observations are convoluted to calculate DDLT and a set of parametric distributions is fitted to the demand data. Our candidate distributions for DDLT are beta, gamma, normal and lognormal, for which we use their density function to calculate the respective $90 \%$ percentiles followed by rounding to the nearest integer demand value. This enables the calculation of the percentage error between these values and the empirical $90 \%$ percentile. Given the strong and stable performance of the lognormal distribution, especially when the variance of the lead time is high, we opt for this type of distribution to approximate the DDLT pattern.

Next, we derive the two parameters $\beta_{r}$ and $\gamma_{r}$ that describe the lognormal distribution at remanufacturing center $r$ as

$$
\begin{aligned}
& \beta_{r}=\ln \left(\frac{\hat{\mu}_{D D L T_{r}}}{\left.\sqrt{\frac{\hat{\sigma}_{D D L T_{r}}^{2}}{\hat{\mu}_{D D L T_{r}}^{2}}}\right)}\right) \\
& \gamma_{r}=\sqrt{\ln \left(\frac{\hat{\sigma}_{D D L T_{r}}^{2}}{\hat{\mu}_{D D L T_{r}}^{2}}+1\right)}
\end{aligned}
$$

with $\hat{\mu}_{D D L T_{r}} \approx \lambda_{r}^{\prime} L T_{r}$ (Little's law) and $\hat{\sigma}_{D D L T_{r}}^{2} \approx \sigma_{D_{r}}^{2} L T_{r}+\sigma_{L T_{r}}^{2}\left(\lambda_{r}^{\prime}\right)^{2}$, and where $\sigma_{D_{r}}^{2}$ is the variance of the demand for critical parts at facility $r$, i.e. parts for overhauls delivered from stock located at the outbound of facility $r$. To obtain this value, we have to add the independent demand variances of all the parts that leave facility $r$, or $\sigma_{D_{r}}^{2}=\sum_{c=1}^{C} \sum_{s=1}^{S-1} \sigma_{D_{c s r}}^{2}$. We know that parts for machines with service type $s$ in region $c$ are demanded according to a triangular distribution with parameters $e_{c s}, f_{c s}$ and $g_{c s}$ and that some net fraction 
$\tau_{c s r}\left(1-\omega_{c s r}\right)$ is satisfied by facility $r$. The demand for this net fraction is also triangularly distributed with parameters $e_{c s r}=\tau_{c s r}\left(1-\omega_{c s r}\right) e_{c s}, f_{c s r}=\tau_{c s r}\left(1-\omega_{c s r}\right) f_{c s}$ and $g_{c s r}=\tau_{c s r}\left(1-\omega_{c s r}\right) g_{c s}$. So we have $\sigma_{D_{c s r}}^{2}=\left(e_{c s r}^{2}+f_{c s r}^{2}+g_{c s r}^{2}-e_{c s r} f_{c s r}-e_{c s r} g_{c s r}-f_{c s r} g_{c s r}\right) / 18$. Next, we can determine the value of $S_{r}$ by using the $x_{r} \%$ quantile of the lognormal distribution

$$
q \operatorname{lnorm}\left(x_{r} \%, \beta_{r}, \gamma_{r}\right)=\left\lfloor e^{\beta_{r}+z\left(x_{r}\right) \gamma_{r}}+0.5\right\rfloor
$$

with $z\left(x_{r}\right)$ obtained from the standard normal table. Note that $S_{r}$ is also equal to $\hat{\mu}_{D D L T_{r}}+\hat{S S_{r}}$, so we have $\hat{S S}_{r}=q \operatorname{lnorm}\left(x_{r} \%, \beta_{r}, \gamma_{r}\right)-\hat{\mu}_{D D L T_{r}}$. A similar approach is used for production plant $n$.

This $S$-level ensures that parts can be delivered from stock with given probability. In order to obtain the expected number of units in inventory at remanufacturing $\left(E N_{c s r}\right)$ and manufacturing $\left(E N_{c s n}\right)$, including transportation, we rely on Little's Law: expected work-in-process equals expected arrival rate multiplied by expected lead time. Since scrap in the remanufacturing process leads to higher arrival rates $\lambda_{c s r}$ than the rates used in $\hat{\mu}_{D D L T_{r}}$, total average work-in-process can exceed this value. So we have for $x=r$ and $x=n$

$$
E N_{c s x} \approx \lambda_{c s x} L T_{c s x}
$$

Finally, total inventory costs in the network is the sum of work-in-process and safety inventory valued at their unit holding costs $H C_{x}$, the cost to keep one unit in stock during a year at the corresponding operation $x$

$$
\begin{aligned}
I C \approx & \left.\sum_{r=1}^{R}\left(\sum_{c=1}^{C} \sum_{s=1}^{S-1} H C_{c s r} E N_{c s r}+H C_{r} \hat{S S}\right)_{r}\right)+ \\
& \sum_{n=1}^{N}\left(\sum_{c=1}^{C} \sum_{s=1}^{S-1} H C_{c s n} E N_{c s n}+H C_{n} \hat{S S_{n}}\right)
\end{aligned}
$$




\subsection{Profit Model Optimization}

The mathematical model determines the optimal network structure and the optimal contract design (price and uptime guarantee). The objective function is profit maximization. The decision variables are $Y_{r}$ (location decision), $s_{t}$ and $s_{r}$ (capacity decision), $\varpi_{c s}$ (demand volume decision), $T O_{1}(F R C$ overhaul interval decision) and $\tau_{c s r}$ (flow resource assignment decision).

Equations 3, 6, 7, 8 and 19 can be combined into an overall profit objective function to characterize all the trade-offs described in the problem formulation

$$
\text { Profit } \approx R E V-F C-T C-P C-I C
$$

This function needs to be maximized subject to Constraints 5 and 11 , logical constraints $Y_{r} \in\{0,1\}$, and boundary constraints for each decision variable: $s_{t}^{\min } \leq s_{t} \leq s_{t}^{\max }, s_{r}^{\min } \leq s_{r} \leq s_{r}^{\max }, 0 \leq \varpi_{c s} \leq 1$, $T O_{c s}^{\min } \leq T O_{c s} \leq T O_{c s}^{\max }$ and $0 \leq \tau_{c s r} \leq 1$. This optimization is hard because it consists of several difficult sub-problems with a non-linear objective function, non-linear constraints and mixed integer variables. Furthermore, there are various conditional relationships in the correction factor $\phi$. Although a heuristic search approach can never guarantee global optimality, it is a search method that is suitable to handle our difficult mixed integer non-linear programming (MINLP) problem. Population-set direct search based methods are commonly used to optimize multi-modal problems with non-linear, non-differentiable and non-smooth objective functions. These methods do not require any properties of the objective function. Within the broad family of search algorithms we opt for a genetic algorithm, more specifically a Differential Evolution (DE) algorithm. According to Lampinen and Zelinka (1999), DE outperforms any of the competing heuristic methods like branch \& bound using sequential quadratic programming, integerdiscrete-continuous non-linear programming, simulated annealing, non-linear mixed-discrete programming, etc., also for hard non-linear objective functions with multiple non-trivial constraints. Similarly, Ali and Törn (2004) have demonstrated that DE-type algorithms are more robust than other genetictype algorithms because all population members are possibly updated after each generation. In this way DE maintains a better balance between intensifying and diversifying current solutions in the population, which results in a more widely exploration of the search space. Furthermore, DE has proven to perform 
strongly for MINLP network design models (Lieckens et al., 2012). Successful usage of DE-type algorithms in other areas with difficult functions, like scheduling (Damak et al., 2009), neural networks (Piotrowski et al., 2012), frequency assignment in satellite communication systems (Damak et al., 2009), biocompatible solvent design (Cheng and Wang, 2007), etc. further motivates our choice.

Briefly summarized, DE creates an initial population of different solution members. Each member contains a value for each decision variable, randomly selected between their lower and upper bounds. Since many $\tau_{c s r}$ variables are involved, we have observed that it takes a long time before the constraint handling procedure results in feasible solutions that satisfy the inequality constraints 5 . Therefore, we suggest to generate the initial values for the $\tau_{c s r}$ variables randomly between zero and a temporary upper bound of $1 / R$, immediately followed by resetting it to the default value of one for subsequent generations. The other inequality constraints 11 are handled by the procedure as described in Lampinen (2001). The next step is to build children with a mutation and recombination process that takes the difference of randomly sampled population members. Instead of using only local information for each population member, DE mutates all these members with the same universal distribution. In this way, the whole search space is covered which provides more certainty that the found solution is not a local optimum. Another advantage of DE for problems with integer variables, is the use of continuous values to create subsequent children, followed by rounding them down each time the objective function is evaluated. This procedure avoids sub-optimal results in two ways: only feasible solutions give feedback to the optimization process and the risk of premature convergence is lower because of a more diversified population.

As suggested in Lieckens et al. (2012), another option to avoid sub-optimal solutions is to solve the integrated model that treats the four sub-models simultaneously. We have frequently observed that a sequential approach leads to unsatisfactory results. All the strategic questions are not only interrelated (e.g. inventory investments depend on waiting times, which depend on network structure and capacity levels; selling prices depend on volume and downtime, which depend on overhaul frequency and technician waiting time; etc.), better solutions can also be found by intermediate values for overhaul frequency and volume assignments. Nevertheless, extensive scenario computations reveal that the binary decision variables $Y_{r}$ pose the largest burden on the escape from local solutions, especially when the profit function is rather flat for distinctive network layouts. In order to overcome this problem, we decompose the decision 
variables into two subsets: one set for the binary variables and one set for all the other decision variables. This is implemented in the DE algorithm by using two separate populations, one for each subset, where DE mutation and recombination processes are performed completely independent of each other. When both populations have converged to solutions with precision $\delta$ between the best and worst member, the search algorithm is terminated. This approach can also be found in Neri and Tirronen (2010).

Although different, complex mutation schemes exist (Das, 2011), we opt to handle both populations by the basic and commonly used $D E / r a n d / 1 /$ bin scheme with crossover constant $C R=0.9$, mutation scaling factor $F=0.6$, population size of twice the number of decision variables, and the maximum number of generations equal to infinity. The reason is that we are only interested in the best solutions, which can only be found when all the population members have converged to approximately the same objective value with precision $\delta=10^{-5} \%$. Since the goal of the paper is not to find the best DE scheme with the best parameter setting regarding the computation time, testing other schemes with different control parameters values is out of our research scope. We refer to Lieckens and Vandaele (2007) for more details about this algorithm.

\section{Case Study Results}

In this section we discuss the insights derived from a computational experiment testing multiple scenarios. The base case scenario is defined based on cost, time and reliability data of AirGen, but to find appropriate demand sensitivities we have tested multiple values. The demand sensitivity values that lead to realistic results for the situation at AirGen are selected as the base case scenario. Hence, the base case scenario represents the situation at AirGen. Next, different scenarios in which the AirGen parameter values are increased and decreased are solved in order to obtain more general insights. The scenarios vary in terms of demand sensitivities for price $\left(\varepsilon_{S P_{c}}\right)$ and downtime $\left(\varepsilon_{D T_{c}}\right)$, labor costs (yearly wage cost of remanufacturing operators $\left(F C 2_{r}\right)$ and field technician $\left.\left(F C 2_{t}\right)\right)$, machine reliability $(\alpha, \beta)$, price and downtime of the no-service option ( $S P_{c 3}$ and $D T_{c 3}$, respectively). Although confidentiality concerns do not allow a detailed presentation of the base case results we first briefly discuss these results. Next, more general insights are presented. 
The base case scenario is characterized by cost and time parameters of AirGen and demand sensitivity values equal to $-0,004$ and $-0,025$ for price $\left(a_{S P}\right)$ and downtime sensitivity $\left(a_{D T}\right)$, respectively. Moreover, also the cost and downtime of the do-it-yourself option (or no service option) has to be determined if we want to characterize customer choices. We assume that customers that do not opt for service by AirGen bear a yearly cost of 2300 euro, with 100 hours of downtime a year. Based on these parameters the following solution is found: it is optimal for AirGen to sell the full responsibility contract (FRC) at a yearly price that is considerably higher than the cost of the no service option. However, customers that buy ad hoc service still spend more money than those buying a service contract. Moreover, customers with a service contract enjoy a high machine uptime guarantee (only 49 hours of yearly downtime), while those with ad hoc service experience 79 hours of downtime a year. Consequently, most customers that buy service from AirGen opt to buy a FRC. The optimal logistics network has two open remanufacturing facilities.

Starting from the base case scenario different scenarios are created and solved. Instead of discussing the detailed results we present some general insights. First, we explore in which scenarios it is most profitable to offer aftermarket services. Secondly, we highlight the principal determinants of the service contract price and uptime guarantee. Furthermore, we discuss the results of scenarios in which the OEM faces two well known problems of selling comprehensive service contracts, i.e. adverse selection and high labor costs. Lastly, our results reveal the importance of taking into account the endogenous character of the replenishment lead time when optimizing the logistics network.

The profitability of providing maintenance services strongly depends on the demand sensitivities of customers with respect to price and downtime. To test which customer segments are most profitable, scenarios with different market sensitivities for price $\left(\varepsilon_{S P_{c}}\right)$ and uptime guarantees $\left(\varepsilon_{D T_{c}}\right)$ are tested to assess the effect of targeting different types of customers. The price sensitivities range from low $\left(\varepsilon_{S P_{c}}=-0,0001\right)$ to high sensitivity $\left(\varepsilon_{S P_{c}}=-0,0013\right)$, while at the same time the downtime sensitivities range from low $\left(\varepsilon_{D T_{c}}=-0,0125\right)$ to high sensitivity $\left(\varepsilon_{D T_{c}}=-0,0625\right)$. Customers with a low price sensitivity and a high downtime sensitivity are the most profitable to target. Low price sensitivity allows for charging a premium given superior uptime guarantees (lower downtime): there is a strong sales price increase for FRC, while this is less pronounced for ad hoc interventions. If the downtime sensitivity is high, a small 
improvement in the uptime guarantee is rewarded with a high potential price increase, especially if customers are price insensitive. Hence, the highest uptime guarantee for FRC is given when customers are downtime sensitive and price insensitive. To realize the higher uptime, additional investments in field and remanufacturing capacity are made.

Apart from the demand sensitivities, the downtime of the no service option $\left(D T_{c 3}\right)$ greatly affects both profit and the number of FRC sold. The higher the downtime for customers not buying service from AirGen, the higher AirGen's profits and volume of sold FRC. Surprisingly, a higher downtime for the no service option does not results in lower uptime guarantees. However, it is optimal for the service provider to increase its price for both FRC and ad hoc service, while keeping delivered downtime constant. Although selling prices for FRC and ad hoc increase, more customers choose to acquire service from AirGen. Together with the increased prices, the higher sales volumes result in higher profits. Hence, there appears to be a strong incentive to target customer/machine segments that are difficult to maintain without the necessary expertise that AirGen has, i.e. very complex machines or customers without technical staff. Moreover, this observation implies that equipment manufacturers have a strong incentive to adapt the machine design to suit their own maintenance operations but impede quick maintenance by competing organizations e.g. through build-in diagnostics. Moreover, higher downtime for the no service option results in higher remanufacturing volumes within the AirGen organization, which justifies opening additional remanufacturing facilities. When the cost of maintaining the equipment without AirGen's service is increased (i.e. $S P_{c 3}$ ) instead of increasing the downtime of the no service option, the effects are similar but less pronounced: higher prices result in more profit for AirGen. Nevertheless, it is remarkable that even though the volume of both FRC and ad hoc service increases due to more downtime in the no service option, an increase in the cost of the no service cost leads to more FRC sales but less ad hoc volume (both FRC and ad hoc prices increase).

With respect to the level of centralization in the network we notice that the number of remanufacturing facilities decreases if customers are price and/or downtime sensitive. This tendency is even strong enough to close some of the remanufacturing facilities while the total remanufacturing volume increases. Note that in the scenarios with downtime sensitive customers the opening of less remanufacturing facilities is combined with higher investments in field capacity. When customers are sensitive for price but not for 
downtime, the time between overhauls is lengthened.

The optimal FRC price depends primarily on the price sensitivity (negative correlation) and the downtime of the no service option (positive correlation). Hence, segmenting customers based on their price sensitivity is advised to differentiate prices. Contrary, the uptime guarantee is fairly stable except if the reliability of the machines differs from the base case scenario. With more reliable machines, AirGen is inclined to offer a higher uptime while unreliable machines create a tendency towards lower uptime guarantees. Thus, while price discrimination between customer segments seems advisable, uptime guarantees are preferably based on the machine type and reliability. Moreover, machine reliability characteristics, especially the rate of reliability degradation $(\beta)$, strongly influence the optimal overhaul interval: faster reliability degradation mandates shorter overhaul intervals.

Adverse selection (and/or moral hazard) are well-known problems when selling comprehensive service contracts. We induce these phenomena by defining a scenario in which the reliability of machines under FRC is lower than without FRC, i.e. machines with a contract mandate more service interventions than machines without a contract. As a consequence of adverse selection and the uptime guarantee, AirGen is required to shorten the overhaul interval and invest in more capacity (both remanufacturing operators and field technicians). Moreover, costs are further increased due to the opening of more remanufacturing facilities (and consequently resulting in a more decentralized network compared to the base case). Despite the additional efforts, the downtime is shooting up: AirGen decreases the uptime guarantee it offers in the contracts affected by the moral hazard. This deterioration in service guarantee puts a downward pressure on the price of the contract, while the total volume of sold contracts tumbles. Interestingly, the moral hazard plaguing FRC also puts a negative price pressure on the ad hoc service, which experiences increased sales due to the reduced price and worsened FRC offering. As a result of these increased ad hoc sales, the negative impact on revenues and profits is partly countered. Nevertheless, the adverse selection problem overall results in less revenues, less contracts and reduced profits.

Aftermarket services are typically sold in mature markets with a substantial installed base. However, these markets are often characterized by high labor costs which could threaten the profitability of labor intensive maintenance services. When customers are not price sensitive, labor costs do not have a big impact on the network structure, the selling prices nor profit. However, as customers become more price 
sensitive labor costs become very important. High labor costs with price sensitive customers render remanufacturing operations cost inefficient. Hence, in these scenarios it is profit optimal to deliver newly produced parts instead of remanufactured parts. Moreover, if customers are price sensitive, higher labor costs cause the prices to increase only slightly, but the OEM will mainly lower the service level he provides (lower uptime guarantees for both FRC and ad hoc service). This redesign of the service contracts results in a lower percentage of the installed base covered by service contracts and a strongly reduced profitability of aftermarket services.

Lastly, our model reveals that the commonly accepted paradigm that higher holding costs advocate centralization does not always hold because centralization decisions may lengthen the replenishment lead time (as is the case in our setting). Since lead times influence the required safety stock, shortening these lead times in a decentralized layout can be a valid alternative for centralization. In our case study this is exactly what is observed in high volume scenarios: if the inventory holding costs are increased, the optimal network is more decentralized than before. Opening more facilities reduces the transportation times and hence the inventory replenishment lead time is shortened. With high inventory holding costs, lead time reduction through decentralization can be more cost effective than inventory pooling by centralization.

\section{Conclusions}

In this research we study the interaction between comprehensive service contracts and the logistics network to support the maintenance business. We formulate a mixed integer non-linear problem to optimize the contract and network design with a profit objective. The contract design is determined by its price, uptime guarantee and overhaul interval, while the network design is concerned with the capacity of field technicians, the locations and capacity of remanufacturing facilities, and the inventory levels. We use a multi-echelon queueing model with endogenous demand, which depends on pricing and machine uptime characteristics of three service options, i.e. comprehensive service contracts (FRC), ad hoc service and no service.

Our results show that targeting price insensitive customers is the most profitable strategy, especially 
when they are sensitive to downtime. Long downtimes that customers will experience during failures in the absence of a contract (no service option) also have a strong positive impact on both profit and contract selling. The optimal price for FRC primarily depends on the downtime of the no service option and the price sensitivity of the customers. Hence, price differentiation based on customer characteristics is an appropriate strategy. However, we find that the uptime guarantee is preferably set based on machine characteristics regarding its reliability rather than customer characteristics.

When offering FRC it is important to take measures that avoid adverse selection. Adverse selection results in higher costs caused by more service interventions, shorter overhaul intervals, more capacity investments and a more decentralized network design. As a consequence the price of the FRC needs to be increased and the uptime guarantee is lowered. This results in a lower volume of FRC, less revenues and lower profits. It is clear that adverse selection is a real risk to the profitability of aftermarket services. A second challenge when offering comprehensive service contracts are high labor costs. Our results show that it is possible to sell aftermarket services profitably while facing high labor cost but only when customers are not too price sensitive.

It is important to know that the contract characteristics and the corresponding volumes influence the optimal network. For example providing better service through a higher uptime guarantee requires higher investments in field capacity. Interestingly, we observe that high labor costs in combination with price sensitive customers promotes the production of more new parts instead of remanufacturing them. Inventory costs also have an impact on the network and contract design: decentralization, capacity investments and larger overhaul intervals are the characteristics in case of high unit holding costs and high remanufacturing volume. Lastly, we stress the importance of taking into account lead time when making network optimization decisions: lead time reductions can be a more powerful technique to reduce inventory cost than centralization of inventory.

\section{Acknowledgement}

The authors thank the Fund for Scientific Research Flanders (FWO) for the support: project G.0333.10N and the Post Doctoral Research project for Kris Lieckens. 


\section{References}

Ali, M. and A. Törn (2004). Population set-based global optimization algorithms: some modifications and numerical studies. Computers \& Operations Research 31(10), 1703-1725.

Bollapragada, S., A. Gupta, and C. Lawsirirat (2007). Managing a portfolio of long term service agreements. European Journal of Operational Research 182(3), 1399-1411.

Buzacott, J. and J. Shanthikumar (1993). Stochastic Models of Manufacturing Systems. Englewood Cliffs, NY: Prentice Hall.

Cheng, H. C. and F. S. Wang (2007). Trade-off optimal design of a biocompatible solvent for an extractive fermentation process. Chemical Engineering Science 62(16), 4316-4324.

Damak, N., B. Jarboui, P. Siarry, and T. Loukil (2009). Differential evolution for solving multi-mode resource-constrained project scheduling problems. Computers \& Operations Research 36(9), 26532659.

Das, S. (2011). Differential evolution: A survey of the state-of-the-art. IEEE Transactions on Evolutionary Computation 15(1), 4-31.

Haaijer, R., W. Kamakura, and M. Wedel (2001). The 'no-choice' alternative in conjoint choice experiments. International Journal of Market Research 43(1), 93-106.

Hopp, W. and M. Spearman (2000). Factory Physics. New York: The McGraw-Hill Companies.

Kim, S. H., M. A. Cohen, and S. Netessine (2007). Performance contracting in after-sales service supply chains. Management Science 53(12), 1843-1858.

Lambrecht, M., P. Ivens, and N. Vandaele (1998). ACLIPS: A capacity and lead time integrated procedure for scheduling. Management Science 44(11), 1548-1561.

Lampinen, J. (2001, September 10-24). Multi-constrained nonlinear optimization by the differential evolution algorithm. In 6th On-Line World Conference on Soft Computing in Industrial Applications (WSC6). 
Lampinen, J. and I. Zelinka (1999). Mechanical Engineering Design Optimization by Differential Evolution, Chapter 8, pp. 127-146. London: McGraw-Hill.

Lieckens, K. and N. Vandaele (2007). Reverse logistics network design: The extension towards uncertainty. Computers and Operations Research 34(2), 395 - 416.

Lieckens, K. T., P. J. Colen, and M. R. Lambrecht (2012). Optimization of a stochastic remanufacturing network with an exchange option. Decision Support Systems. http://dx.doi.org/10.1016/j.bbr.2011.03.031.

Murthy, D. N. P. and V. Yeung (1995). Modeling and analysis of maintenance service contracts. Mathematical and Computer Modelling 22(10-12).

Neri, F. and V. Tirronen (2010). Recent advances in differential evolution: a survey and experimental analysis. Artificial Intelligence Review 33(1-2), 61-106.

Nozick, L. K. and M. A. Turnquist (2001). A two-echelon inventory allocation and distribution center location analysis. Transportation Research Part E-Logistics and Transportation Review 37(6), 425441.

Pangburn, M. S. and E. Stavrulaki (2008). Capacity and price setting for dispersed, time-sensitive customer segments. European Journal of Operational Research 184, 1100-1121.

Piotrowski, A. P., P. M. Rowinski, and J. J. Napiorkowski (2012). Comparison of evolutionary computation techniques for noise injected neural network training to estimate longitudinal dispersion coefficients in rivers. Expert Systems with Applications 39(1), 1354-1361.

Pongpech, J. and D. N. P. Murthy (2006). Optimal periodic preventive maintenance policy for leased equipment. Reliability Engineering \& System Safety 91(7), 772-777.

Ray, S. and E. M. Jewkes (2004). Customer lead time management when both demand and price are lead time sensitive. European Journal of Operational Research 153(3), 769-781. 
Robotis, A., S. Bhattacharya, and L. N. Van Wassenhove (2012). Lifecycle pricing for installed base management with constrained capacity and remanufacturing. Production and Operations Management 21(2).

Shanthikumar, J. and J. Buzacott (1981). Open queueing network models of dynamic job shops. International Journal of Production Research 19(3), 255-266.

So, K. C. and J. S. Song (1998). Price, delivery time guarantees and capacity selection. European Journal of Operational Research 111(1), 28-49.

Vermeulen, B., P. Goos, R. Scarpa, and M. Vandebroek (2011). Bayesian conjoint choice designs for measuring willingness to pay. Environmental \& Resource Economics 48(1), 129-149.

Whitt, W. (1993). Approximations for the GI/G/m queue. Production and Operations Management 2(2), $114-161$.

Yeh, R. H., K. C. Kao, and W. L. Chang (2009). Optimal preventive maintenance policy for leased equipment using failure rate reduction. Computers \& Industrial Engineering 57(1), 304-309.

Yeh, R. H., K. C. Kao, and W. L. Chang (2011). Preventive-maintenance policy for leased products under various maintenance costs. Expert Systems with Applications 38(4), 3558-3562. 